\title{
EDITORIAL
}

\section{World Molecular Imaging Congress 2016: Imaging Biology-Improving Therapy}

\author{
Fabian Kiessling, ${ }^{1}$ Christopher H. Contag ${ }^{2,3}$ \\ ${ }^{1}$ Experimental Molecular Imaging, University Clinic and Helmholtz Institute for Biomedical Engineering, RWTH Aachen University, \\ Aachen, Germany \\ ${ }^{2}$ Department of Pediatrics, Stanford University, Stanford, CA, USA \\ ${ }^{3}$ Departments of Radiology, Microbiology \& Immunology, Stanford University, Stanford, CA, USA
}

Two decades of molecular imaging research have led to the creation of a vibrant and dynamic scientific community comprised of pioneers who are creating tools to study the biology of living systems and drive therapeutic innovations. During this time, our core technologies have maturated to a point where they are having a real impact on clinical studies and patient care. Therefore, the theme of the World Molecular Imaging Congress 2016 (WMIC) "Imaging Biology-Improving Therapy" is comprised of two important missions of the molecular imaging field: "Imaging Biology" highlights the aim of developing and applying imaging tools that elucidate biological processes and reveal pathophysiology. In this way, diagnostic imaging has transitioned from descriptive and anatomic measures to functional and mechanistic analyses. The 2016 theme emphasizes that it is the biomedical question that drives the development of new tools and more efficient probes and methods. The therapy aspect of this year's theme is the ultimate objective of our research, that is, improving patient outcomes through early diagnosis and guided therapy. We highlight the fact that diagnosis and therapy are closely linked and that earlier and refined diagnosis inform and improve therapeutic intervention and reduce costs. More comprehensive understanding of biological mechanisms will enable the development of novel therapeutic strategies, and detection of biomarkers will help us assign precise treatments for each individual patient.

The thematic underpinnings and structure of our 2016 congress center on biomedical problems, and are based on a matrix structure with the biology on one axis and the technology on the other axis. The vertical axis of the matrix contains the five major biomedical emphases of oncology, neuroscience and inflammation, as well as infectious, metabolic, and cardiovascular diseases. The horizontal axis categorizes them into the research focus areas of probes and targets, preclinical imaging, new biology, and first-in-human and clinical studies". In order to cover areas that cannot easily be assigned to one of these

Correspondence to: Fabian Kiessling; e-mail: fkiessling@ukaachen.de emphases but that may impact several fields, two further emphases are included. One of them we called "instrumentation, computation and omics" and will cover innovations in device engineering such as microscopy, image-guided surgery, computer applications and postprocessing and optogenetics. The other we titled "transdisciplinary imaging" and includes emerging research fields such as imaging stem cells in regenerative medicine and reproductive health, visualization of developmental processes as well as systems biology, genome editing and synthetic biology. This structure was designed to create highly interdisciplinary scientific sessions featuring multimodality solutions to the most pressing questions in biomedicine. It is our intent that this structure will broaden the scope of knowledge for attendees and inspire new collaborations that will lead to new imaging tools and greater understanding of human health and disease.

WMIC 2016 features a dedicated track for scientific contributions from industry, where our exhibitors can compete for the "Innovation of the Year Award". Continuing the success from last year, the best-rated industry innovations will be presented in the exhibition area and the winner selected in real-time by the audience.

The scientific contributions are the heart of our meeting, and we are grateful to all of you who submit abstracts and support the meeting as reviewers, category and emphasis chairs, and those who help us select the contributions for oral presentations. Multiple awards like the Young Investigator Award (YIA), the Aspect Imaging Travel Grant, travel stipends, the "Women in Molecular Imaging Network (WMIN) Scholar Award", and the poster awards acknowledge your outstanding contributions to our field and our meeting. To further highlight excellent contributions, our poster chair Dr. Alexander Klibanov has taken measures to further valorize the poster sessions as an important platform for scientific exchange. In this context, for the first time, we will feature the best-rated posters as oral presentations at the beginning of each poster session. Food and drinks, served during the poster sessions, will further stimulate and catalyze scientific interactions. 
Highlights of the WMIC will be the plenary sessions. They will start with the opening lecture by the president of Memorial Sloan Kettering Cancer Center, Dr. Craig Thompson, on cancer metabolism. With respect to the intersection of cancer diagnosis and therapy, three of our plenary speakers will present data in the emerging field of image guided interventions: Dr. Claire Tempany will speak on MR-guided focused ultrasound surgery, Dr. Chrit Moonen will lecture on hyperthermia and thermobiology, and Dr. Thomas Wang will describe insights gleaned through molecular endoscopy. Another three plenary lectures feature studies in neuroscience with Dr. Karl Deisseroth talking about controlling cell function with light in the field of optogenetics, Dr. Gitte Moos Knudson discussing the measurement of neurotransmitter release, and Dr. Larry Wald demonstrating tools for understanding the brain in a technology-oriented lecture. Dr. Tian Xu will present his work on developing in vivo genome screens for gene discovery in living animals. Finally, Dr. Manu Prakash will talk about how curiosity-driven research led his group to develop origami-like paper microscopes and other curious tools. Furthermore, to ensure that all the participants hear about all the important contributions, we are very grateful to Dr. Thomas J. Meade, who agreed to summarize the most innovative contributions to the conference in a "highlight plenary".

Twelve spotlight sessions reflect major research areas of the WMIS and accentuate areas with anticipated importance to the molecular imaging field. There will be exciting spotlight sessions showing recent trends and developments in imaging tumor heterogeneity, cardiovascular diseases, metabolic diseases, infections, and inflammation. The translation from preclinical to clinical evaluation including the use of large animal models will be demonstrated in the spotlight sessions in the areas of translational neuroscience and neurodegenerative diseases and optoacoustic clinical translation. Additionally, there will be an important and highly informative session about imaging for patient stratification in clinical studies. Attendees are further recommended to attend exciting talks given in the spotlight sessions highlighting ultrasound molecular imaging and drug delivery, and another about imaging in reproductive health and stem cell biology, which will also have a significant translational component. We hope to stimulate research activity aimed at visualizing the growth of new tissues and organs with a spotlight session on tissue engineering and regenerative medicine. Furthermore, the spotlight sessions on "synthetic biology" and "in vivo gene screens and genome editing" will stimulate new directions in the field of molecular imaging. Finally, our "Early Stage Professionals in Molecular Imaging Sciences (ESPMIS)" interest group will sponsor a spotlight session on the opportunities and pitfalls in writing grant applications and advancing academic careers. It is important to acknowledge that all WMIS interest groups were intimately involved in the selection of topics and the recruitment of speakers for the spotlight sessions, and we encourage all of you to get involved with the WMIS interest groups. We are grateful to the "European Society for Molecular Imaging (ESMI)", the "Federation of Asian Societies for Molecular Imaging
(FASMI)", and the "National Institutes of Health (NIH)" for helping to put together outstanding spotlight sessions.

The first day of the conference will again be dedicated to education. Dr. Danielle Vugts and her co-chair Dr. Charles Manning put in tremendous effort in composing an outstanding educational program. In this context, they followed the new concept that was introduced during the 2015 WMIC meeting in Hawaii to link basic education to our focus areas. The NIH, the "American Association of Physicists in Medicine (AAPM)", and the "Society of Nuclear Medicine and Molecular Imaging (SNMMI)" assembled sessions that address hybrid imaging, immuno-PET, hyperpolarized MRI, pH-sensing, and point-ofcare molecular diagnostics. The educational sessions organized by the "Managers of Molecular Imaging Laboratories (MOMIL)" and ESPMIS interest groups complement these sessions by addressing the skills needed to run successful imaging programs. In these sessions, speakers will discuss questions, which are crucial for our young but certainly also many advanced researchers like how to run a laboratory, how to bring new probes and methods into clinical use, and how to initiate a start-up company. As a community and society, we recognize the important role that corporations play in advancing imaging tools and making them user-friendly, and acknowledge their critical role in clinical translation. We are grateful to have such incredible corporate sponsors of our program.

The future direction of molecular imaging will be discussed in the fellows forum, where two pioneers of our society, Dr. Johannes Czernin and Dr. Ralph Weissleder, will share their thoughts and experiences. They will address the provocative questions of "Can imaging ever make medicine more precise?" and "What is the role of imaging in systems biology?"

The WMIC has been the place for scientific exchange in molecular imaging and offers an excellent venue to meet old friends and build your professional network. Therefore, in addition to the conference reception on the first day, and the "Young Investigator Cocktail Hour", you can take advantage of the proximity of the meeting venue to Central Park, many museums, bars, and restaurants and explore the fascinating city of New York. The gala event winds up the meeting with a celebration at a beautiful and very typical New York venue.

If you did not get your abstract in by the March deadline, do not worry! There will be a late-breaking abstract submission opportunity from May 25 to June 15, 2016. Therefore, we welcome submission of your hottest new abstract to the World Molecular Imaging Congress 2016-it is not too late to feature your work at the premier meeting in multiscale and multidisciplinary molecular imaging. The World Molecular Imaging Society is looking forward to welcoming you in New York!

\section{Compliance with ethical standards}

\section{Conflict of interest}

The authors declare that they have no conflicts of interest. 